\section{PS7:142 LUPUS LOW DISEASE ACTIVITY STATE (LLDAS) DEFINITION IN A MONOCENTRIC SYSTEMIC LUPUS ERYTHEMATOSUS PATIENT COHORT AND ITS CORRELATION TO ORGAN DAMAGE}

A Lladó, A Taulaigo, M Vicente, MF Moraes-Fontes. Unidade de Auto-Imunes, Serviço de Medicina 2, Hospital Curry Cabral, Centro Hospitalar de Lisboa Central, Lisbon, Portugal

10.1136/lupus-2018-abstract.185

Purpose Lupus Low Disease Activity State (LLDAS) was defined and validated in 2016 by a panel of lupus experts. When attained, it seems to be associated with prediction of clinical improvement and allow a treat-2-target (T2T) approach in clinical care. LLDAS definition was applied to the cohort of SLE patients followed at our Autoimmune Disease Unit and correlated with damage accrual.

Methods Demographic, clinical and immunological features were recorded at baseline. Data were prospectively collected from January 2013 to July 2017. At each consultation during the study period, disease activity, current therapy and fulfilment of LLDAS were registered, except for the Physician Global Assessment which was not recorded. Organ damage progression was evaluated by SLICC damage index at inclusion and at the last evaluation. Spearman's rho test was used, with $\mathrm{p}<0.05$ considered statistically significant (SPSS Statistics, version 23.0).

Results 76 patients were included: 93.4\% females, 88.2\% Caucasian, mean age and mean disease duration at inclusion 45.9 \pm 13.3 and $14.0 \pm 8.3$ years, mean of follow-up at recruitment of $9.4 \pm 5.1$ years. Overall, 1043 visits were performed. As regards LLDAS achievement, $90.8 \%$ of patients were in LLDAS at least in $25 \%$ of the time, $76.3 \%$ at least in $50 \%$, $55.3 \%$ at least in $75 \%, 31.6 \%$ at least in $90 \%$ and $15.8 \%$ for the entire follow-up. At the last observation 33 patients (43.4\%) were on treatment with glucocorticoids, $42.1 \%$ had their dose reduced during the study and $86,8 \%$ were under a dose of $7.5 \mathrm{mg}$ daily; 8 patients were taking belimumab, 2 rituximab and 2 cyclophosphamide. Median SLICC at onset and last visit were 0 and 1 , respectively, and IQR SLICC was the same (IQR, -1.5-2.5). The time in LLDAS was associated to less number of global flares (CC $-0.541, \mathrm{p}<0.001)$ but no correlation was found with organ injury (CC -0.013 , $\mathrm{p}<0.911$ ).

Conclusions Majority of our patients were in LLDAS during the follow-up period of 4.5 years. LLDAS was associated with less global flares, but not with reduced organ damage. Further studies are important in order to conclude if these targets could be attained more actively with T2T approaches.

\section{PS7:143 SEVERITY ASSESSMENT OF LUPUS PATIENTS: DATA FROM THE LUPUS REGISTRY IN CRETE, GREECE}

\footnotetext{
1,2 Gergianaki, ${ }^{3} \mathrm{~A}$ Fanouriakis, ${ }^{1} \mathrm{~A}$ Repa, ${ }^{1} \mathrm{C}$ Adamichou, ${ }^{1} \mathrm{M}$ Terizaki, ${ }^{1} \mathrm{G}$ Spyrou, ${ }^{1,2} \mathrm{P}$ Sidiropoulos, ${ }^{2,3,4} \mathrm{D}$ Boumpas, ${ }^{1,2} \mathrm{G}$ Bertsias. ${ }^{1}$ Rheumatology, Clinical Immunology and Allergy, University of Crete School of Medicine, Iraklio, Greece; ${ }^{2}$ Institute of Molecular Biology-Biotechnology, FORTH, Iraklio, Greece; ${ }^{3} 4$ th Department of Medicine, Attikon University Hospital, University of Athens Medical School, Athens, Greece; ${ }^{4}$ Biomedical Research Foundation of the Academy of Athens, Greece
}

\subsection{6/lupus-2018-abstract.186}

Purpose To provide an updated, comprehensive assessment of the burden and severity of SLE manifestations at the community level.
Methods Data were retrieved from the Cretan Lupus Registry, which includes adult patients with SLE who are regularly followed in Crete. SLE is defined as mild, moderate or severe, based on the BILAG glossary of the severity of disease manifestations and the use of potent immunosuppressive drugs. Organ damage was assessed through the SLICC/ACR Damage Index [SDI].

Results A total 737 SLE patients (98\% with $\geq 4$ ACR-1997 criteria, 74\% fulfilling both ACR-1997 and SLICC-2012 criteria) were included in the present analysis, with a median (interquartile range) age at diagnosis of 43 (21) years and disease duration of 8 (7) years. Regarding disease severity, 49\% of the patients had mild, 32\% moderate and 19\% severe lupus. Within the severe cases, most frequently afflicted systems were the haematological (4.6\%), renal (3.6\%), cardiovascular system (2.8\%), and neurological (2.3\%). Mycophenolate was administered in $0 \%, 2.7 \%$ and $8.5 \%$, and rituximab in $0 \%, 7.1 \%$ and $15.3 \%$ of patients with mild, moderate and severe disease, respectively $(\mathrm{p}<0.01$ for both). Disease severity did not differ according to age of SLE diagnosis (before or after 50 years), whereas female predominance was more pronounced in mild cases $(31: 1)$ as compared to moderate $(12: 1)$ or severe $(5: 1) \quad(p<0.001)$. Notably, more severe disease correlated with shorter time interval from symptoms onset to SLE diagnosis (delay $>12$ months: $56 \%$ in mild, $39 \%$ in moderate, $27 \%$ in severe, $\mathrm{p}<0.001)$. Unemployment and smoking status ( $n=399$ patients) tended to be higher in the moderate/severe group (54\% versus $44 \%$ and $34 \%$ versus $28 \%$, respectively). Regression analysis showed that moderate/severe as compared to mild disease is strongly associated (odds ratio 2.5, $\mathrm{p}<0.001)$ with organ damage accrual (SDI $>0)$.

Conclusions At the community level, more than half of SLE patients present with moderate or severe manifestations which may contribute to irreversible organ damage.

\section{PS7:144 APPLICATION OF THE DORIS ALGORITHM FOR THE DEFINITION OF DISEASE REMISSION OVER A 2-YEAR PERIOD IN A COHORT OF ITALIAN PATIENTS WITH SYSTEMIC LUPUS ERYTHEMATOSUS}

${ }^{1} \mathrm{~F}$ dall'Ara, ${ }^{1} \mathrm{~L}$ Andreoli, ${ }^{1} \mathrm{~F}$ Migliorati, ${ }^{1} \mathrm{G}$ Armentaro, ${ }^{1} \mathrm{M}$ Fredi, ${ }^{1} \mathrm{M}$ Frassi, ${ }^{1} \mathrm{~F}$ Franceschini, ${ }^{2} S$ Calza, ${ }^{1}$ A Tincani. ${ }^{1}$ Rheumatology and Clinical Immunology, Department of Clinical and Experimental Sciences, Spedali Civili and University of Brescia, Italy; ${ }^{2}$ Biostatistics Unit, Department of Translational and Molecular Medicine, University of Brescia, Italy

\subsection{6/lupus-2018-abstract.187}

Objective Systemic Lupus Erythematosus (SLE) is characterised by a fluctuating course.To achieve sustained remission is the goal of maintenance treatment. In 2014, an international Task Force named DORIS proposed four definitions of remission. Aim of this study was to evaluate the performance of the DORIS algorithm in comparison to the remission status as defined by clinical judgement.

Methods Monocentric retrospective study. Among all SLE patients followed at the Lupus Clinic between 2014 and 2016, we enrolled patients fulfilling the SLICC 2012 criteria who were visited at least once in 2016 and who had at least 5 biannual medical examinations in the previous 2 years. Remission according to DORIS was defined as a clinical-SLEDAI (cSLEDAI) score equal to zero and Physician Global Assessment $(\mathrm{PGA})<0.5$. Remission Off treatment: corticosteroids and immunosuppressant-free patients with antimalarials allowed; Remission On treatment: antimalarials and/or daily 